\title{
ULTRASONIC ATTENUATION DUE TO THE NEUTRAL ACCEPTOR Mn IN GaAs
}

\author{
K. Laßmann and Hp. Schad \\ Max-Planck-Institut für Festkörperforschung, Heilbronnerstrasse 69, 7000 Stuttgart 1, Deutschland
}

(Received 28 July 1975 by M. Cardona)

\begin{abstract}
We report on measurements of the ultrasonic attenuation in GaAs: $\mathrm{Mn}$ at frequencies between 400 and $2000 \mathrm{MHz}$ and at temperatures between 1 and $35 \mathrm{~K}$. The results indicate that there is a level $3 \mathrm{meV}$ above the acceptor ground state.
\end{abstract}

PHONONS are effectively scattered by bound electrons and holes in cubic semiconductors as found by measurements of thermal conductivity, ultrasonic attenuation and experiments with heat pulses and superconducting tunnel junctions. However, not all the results are well understood by theory, especially in the case of ultrasonic attenuation due to neutral acceptors. This may be due to the fact that the experimental conditions are not always well-defined, as was pointed out by Ishiguro: ${ }^{1}$ wave function overlap at high concentration of shallow acceptors, unknown distribution of internal strains and electric fields due to dislocations and impurities, uncontrolled strains from transducers cemented to the sample, magnetic field effects in the case of magnetostrictive transducer films.

So far, only shallow acceptors have been investigated by ultrasonic experiments. We present here an extension of earlier measurements on a deeper acceptor, namely GaAs: $\mathrm{Mn}^{2}$

In view of the above-mentioned difficulties this system has several advantages: solubility of $\mathrm{Mn}$ and the small extent of the acceptor wave function (Bohr radius about $10 \AA$ ) allow relatively high doping without wave function overlap; the piezoelectricity of GaAs can be used to generate and detect the ultrasound directly without a transducer; charge state and site symmetry of the Mn ion can be checked by EPR.

The measurements were made with two Czochralskygrown crystals (I and II) of different $\mathrm{Mn}$ concentrations added to the melt $\left(1.8\right.$ and $\left.3.8 \times 10^{18} \mathrm{~cm}^{-3}\right)$ and different dislocation densities $\left(5 \times 10^{5}\right.$ and $\left.5 \times 10^{3} \mathrm{~cm}^{-2}\right)$. The content of other impurities was negligible as seen from mass spectroscopic analysis. Luminescence measurements showed the lines typical for GaAs: Mn. The binding energy $E_{a}$ was deduced from Hall effect measurements as $99.8 \mathrm{meV}$ for crystal II. From the data of reference 3 , such a value corresponds to a low content of compensating donors. In addition to the six HFS lines, preliminary ESR showed for both crystals 15 equally spaced small satellites, which might indicate a lowered symmetry of the Mn site, ${ }^{16}$ as well as two big broad lines with $g \div 6.4$ and 2.9 not reported in references 4 and 5.

The ultrasonic attenuation was measured by the pulse-echo method recording continuously with varying temperature the height of several echos. The echo pattern was exponential up to $1 \mathrm{GHz}$. Coupling to the electromagnetic energy was achieved by a broad band tunable low loss $\lambda / 4$ resonator. $^{6}$

Figure 1 shows the temperature dependence of the attenuation of [111] longitudinal sound waves in crystal I for several frequencies. The rise above $20 \mathrm{~K}$ is due to piezoelectric coupling to thermally excited carriers in accordance with the measured conductivity. The attenuation below $20 \mathrm{~K}$ is ascribed to the $\mathrm{Mn}$ acceptor. It is proportional to $\omega^{2} / T$ below about $3 \mathrm{~K}$ and shows a peak near $8 \mathrm{~K}$. Subtracting the part $\propto \omega^{2} / T$ the height of the latter is $\propto \omega$. The temperature dependence of the attenuation for crystal II is nearly identical apart from the difference in the absolute value which corresponds to the difference in Mn content.

To analyze the experimental curves we assume that the fourfold degenerate level is split into two doubly degenerate Kramer's levels due to random local fields. (presumably a splitting of the levels due to interaction of Mn pairs can be discarded, since the measured attenuation is proportional to the $\mathrm{Mn}$ concentration.) Such a system can be treated in the same way as the two level model of glasses. ${ }^{7}$ There are two different processes leading to a temperature dependent attenuation.

(1) Resonance interaction of the ultrasonic wave with level splittings $E=\hbar \omega$. Integrating over the linewidth of the levels and assuming that the distribution of $E$ varies slowly in the frequency (i.e. energy) range measured one obtains ${ }^{7}$

$$
\alpha=\frac{A(E) h}{4 \rho v^{3} k_{B}} D_{r}^{2} \frac{\omega^{2}}{T}
$$

where $\rho$ is the density of the crystal, $v$ the sound velocity, $D_{r}$ an appropriate deformation potential constant 


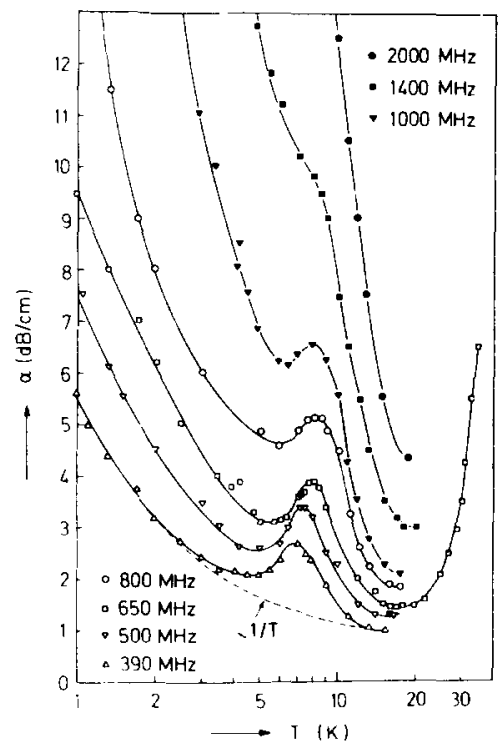

Fig. 1. Ultrasonic attenuation in crystal I for several frequencies as a function of temperature.

and $N(E)$ the spectral density of resonant scatterers. We interpret the low temperature rise of the attenuation as due to such a resonance interaction. To get a rough estimate of the width of the distribution we assume $N(E)$ to be constant up to a maximum value $E_{\max }$ and obtain $E_{\max }=0.04 \mathrm{meV}(\sim h \times 10 \mathrm{GHz})$ taking $D \sim 0.05 \mathrm{eV}^{13}$ Varying the acoustic power in a wide range $\left(10 \mathrm{nW} / \mathrm{cm}^{2}\right.$ to $0.2 \mathrm{~mW} / \mathrm{cm}^{2}$ ) we did not see any saturation effects. ${ }^{7}$

(2) Relaxation of the electronic occupation of split levels with $E \gg \hbar \omega$. The attenuation $\alpha(T)=\int \alpha(E, T) \mathrm{d} E$ is given by the classical formula:

$$
\alpha(E, T)=\frac{C(E, T)}{\tau \bar{\rho} \vec{v}^{3}} \frac{\omega^{2} \tau(E, T)}{1+\omega^{2} \tau^{2}}
$$

where $C(E, T)$ is the difference between relaxed and unrelaxed elastic constants which is given by ${ }^{8}$

$$
C(E, T)=\frac{4 N(E)}{k_{B} T} \frac{\exp \left(E / k_{B} T\right)}{\left[1+\exp \left(E / k_{B} T\right)\right]^{2}} D^{2} .
$$

Three relaxation mechanisms may be envisaged for the system under consideration in determining the relaxation time $\tau(E, T)$

(a) Relaxation due to direct processes

$$
\tau^{-1}(E, T) \propto D^{\prime 2} E^{3} \operatorname{coth}\left(E / 2 k_{B} T\right) f^{\prime}(E) .
$$

Here $D^{\prime}$ is an averaged deformation potential constant and $f^{\prime}(E)$ a form factor describing the reduced interaction with phonons of wavelength smaller than the extent of the acceptor wave function. ${ }^{9}$

(b) Relaxation due to Raman-processes

$$
\tau^{-1}(T) \propto D^{\prime \prime 4}\left(k_{B} T\right)^{5} f^{\prime \prime}(T)
$$

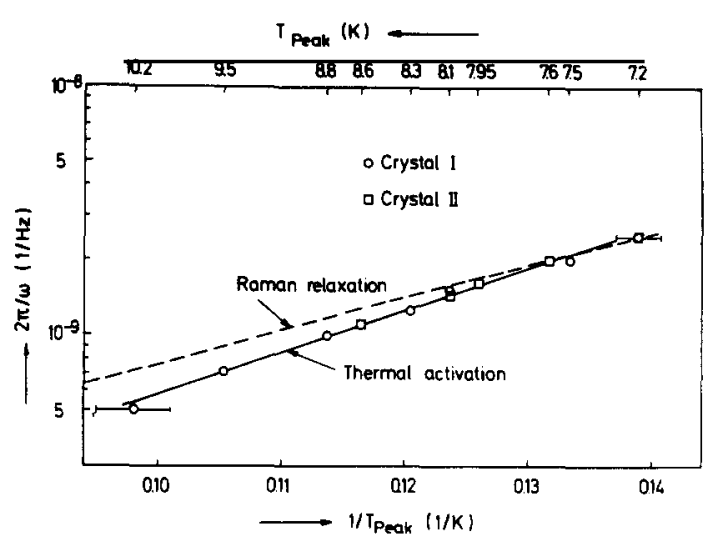

Fig. 2. Frequency dependence of the temperature at which the ultrasonic attenuation peak of Fig. 1. occurs.

where again $f^{\prime \prime}(T)$ is a form factor ${ }^{9}$ reducing the interaction of the Raman phonons at high temperatures (i.e. high energy or small wavelength).

(c) Relaxation due to an Orbach process via an excited level $\Delta \gg E$ above the relaxing levels

$$
\tau^{-1}(T) \propto 1 /\left[1+\exp \left(\Delta / k_{B} T\right)\right] \sim \exp \left(-\Delta / k_{B} T\right)
$$

for $\Delta \gg k T$. The same temperature dependence is obtained for thermal activation over a barrier (Arrhenius equation).

For a comparison with the measured attenuation peak ( $\omega^{2} / T$ subtracted) one should know $N(E)$ which may be rather complicated even when it is due to a regular array of dislocations. ${ }^{10}$ However, $C(E, T) . k T / N(E)$ is a slowly varying function of $E / k T$ as long as $E \lesssim k T$. If this condition holds, a distribution of $E$ will not greatly influence the result in the case of relaxation processes (b) and (c); whereas for direct processes (a) it will tend to broaden the relaxation peak. But even with a single $E$ (whatsoever its value) the calculated relaxation peak is in this latter case much too broad compared to the measured one. The temperature dependence of the peak maximum (approximate condition $\omega \tau(T)=1$ ) is best fitted by an Arrhenius equation for $\tau$ with $\Delta=3 \mathrm{meV}$ while a temperature dependence corresponding to Raman processes describes less well the result (Fig. 2). Furthermore, from the value of the deformation potentials, the absolute value of the Raman relaxation time is too large to attain the condition $\omega \tau(T)=1$ in the measured temperature range.

A "resonance" energy of approximately $3 \mathrm{meV}$ can in fact be deduced from a dip in the thermal conductivity of GaAs: Mn, as first found by Holland ${ }^{11}$ for $\mathrm{Mn}$ concentrations of $5 \times 10^{16}$ and $5 \times 10^{17} \mathrm{~cm}^{-3}$ and also obtained from recent measurements on our crystal II. ${ }^{12}$ Whether such an energy is due to the depth of the acceptor (dynamical Jahn-Teller effect) or is a peculiarity of 


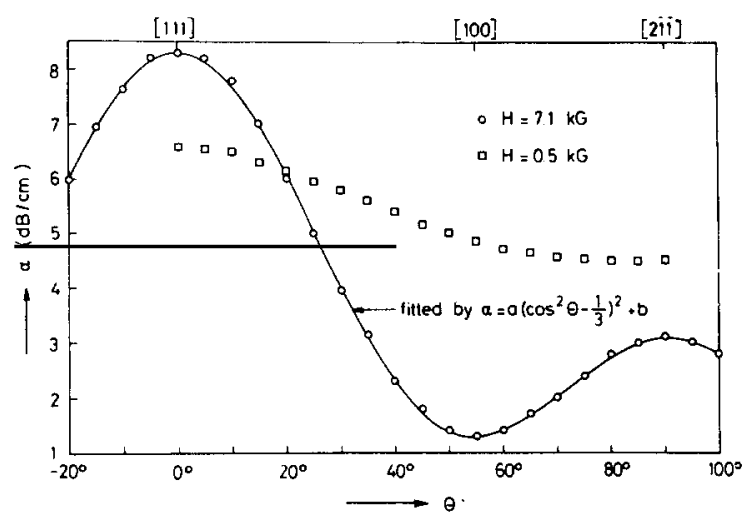

Fig. 3. Angular variation of the attenuation at "high" and "low" fields.

this transition metal (influence of the $3 d$ shell) is not clear. Inserting the recently measured, ${ }^{\mathbf{1 3}}$ strongly reduced deformation potential constants of the Mn acceptor into the condition for stability of a Jahn-Teller effect given by $\mathrm{Bir}^{14}$ it seems that the Jahn-Teller energy is too small compared to the energy of the important phonons of the corresponding vibronic system. Experiments with other deep acceptors may help to clarify the situation. Finally, we should like to mention that the relaxation formula given in reference 9 does not agree with the classical one applied here, and second that none of the relaxation processes discussed here leads (as is sometimes assumed) to an $\omega^{2} / T$-rise of the attenuation for a two-level system.

The magnetic field dependence of the attenuation differs from the results obtained with shallow acceptors. ${ }^{1}$ The main experimental features may be summarized as follows. For both crystals in the entire temperature range below $20 \mathrm{~K}$ and for all orientations of the magnetic field the attenuation changes monotonically for fields up to approximately $2 \mathrm{kG}$ and then remains almost constant up to $7 \mathrm{kG}$ attainable with our magnet and for some special cases measured even up to $70 \mathrm{kG}$. There is a pronounced angular dependence of the attenuation on the magnetic field (Fig. 3) which above $2 \mathrm{kG}$ corresponds to the anisotropy of the squared deformation potential between $\left|m_{j}\right|=\frac{1}{2} \leftrightarrow \frac{3}{2}$ magnetic levels, as calculated from approximate formulas given by Yafet. ${ }^{15}$ With the exception of the peak region for $\theta$ around $0^{\circ}(\theta$ is the angle between [111] and magnetic field directions) the attenuation is lowered by the application of a magnetic field. The peak is not shifted, which means that the relaxation time is independent of the magnetic field in accordance with the angular dependence of the attenuation since in the prefactor $C[E(\theta), T]$ the important dependence on $\theta$ is given by $D^{2}(\theta)$.

In summary we have shown that the ultrasonic attenuation by the relatively deep acceptor $\mathrm{Mn}$ in $\mathrm{GaAs}$ indicates that there is a distribution of level splittings of the ground state (width of the distribution about $0.04 \mathrm{meV}$ ) and an excited state about $3 \mathrm{meV}$ above the ground state. This may be due to a Jahn-Teller effect of the acceptor ground state, but further experiments (e.g. with other deep acceptors) are necessary to get more confirmation about the nature and the existence of such a level.

Acknowledgements - We thank W. Eisenmenger and J. Jäckle for many helpful discussions, N. Stath for carrying out the luminescence measurements and U. Konzelmann for the assistance in making possible the EPR measurements. One of us (Hp. Schad) gratefully appreciates the hospitality of the Forschungslabor Philips, Hamburg, where some preliminary measurements in high magnetic fields could be accomplished. This work has been supported by the Deutsche Forschungsgemeinschaft (SFB 67, Stuttgart).

\section{REFERENCES}

1. ISHIGURO T., Phys. Rev. B8, 629 (1973).

2. LABMANN K., Thesis, Universität Göttingen (1967).

3. WOODBURY D.A. \& BLAKEMORE J.S., Phys. Rev. B8, 3803 (1973).

4. ALMELEH N. \& GOLDSTEIN B., Phys. Rev. 138, 1568 (1962).

5. BLEEKRODE R., DIELEMANN J. \& VEGTER H.J., Phys. Lett. 2, 355 (1962).

6. KINDER H., Thesis, Göttingen (1968).

7. JÄCKLE J., Z. Phys. 257, 212 (1972).

8. ISHIGURO T., FJELDLY T.A. \& ELBAUM C., Solid State Commun. 10, 1309 (1972).

9. SUZUKI K. \& MIKOSHIBA N., Phys. Rev. Lett. 28, 96 (1972).

10. WATTS B.R., J. Phys. C6, 1930 (1972). 
11. HOLland M.G., Proc. Int. Conf. Semicond. Phys. p. 713. Paris (1964).

12. LAßMANN K. \& DE COMBARIEU A. (to be published)

13. SCHAIRER W. \& SCHMID M., Phys. Rev. B10, 2501 (1974).

14. BIR G.L., Sov. Phys. JETP 24, 372 (1967).

15. YAFET Y.J. Phys. Chem. Solids 26, 647 (1965).

16. SEGSA K.H. \& SPENKE S., Phys. Status Solidi (a) 27, 129 (1975). 